\title{
Dual-Modality Image-Guided Surgery of Prostate Cancer with a Radiolabeled Fluorescent Anti-PSMA Monoclonal Antibody
}

\author{
Susanne Lütje ${ }^{1}$, Mark Rijpkema ${ }^{1}$, Gerben M. Franssen ${ }^{1}$, Giulio Fracasso ${ }^{2}$, Wijnand Helfrich ${ }^{3}$, Annemarie Eek ${ }^{1}$, \\ Wim J. Oyen ${ }^{1}$, Marco Colombatti², and Otto C. Boerman ${ }^{1}$ \\ ${ }^{I}$ Department of Radiology and Nuclear Medicine, Radboud University Medical Center, Nijmegen, The Netherlands; ${ }^{2}$ Department of \\ Pathology and Diagnostics, University of Verona, Verona, Italy; and ${ }^{3}$ Department of Surgery, University of Groningen, University \\ Medical Centre Groningen, Groningen, The Netherlands
}

Both radionuclide imaging and near-infrared fluorescent (NIRF) imaging have a high sensitivity to detect tumors in vivo. The combination of these modalities using dual-labeled antibodies may allow both preoperative and intraoperative tumor localization and may be used in image-guided surgery to ensure complete resection of tumor tissue. Here, we evaluated the potential of dual-modality imaging of prostate cancer with the monoclonal antibody D2B, directed against an extracellular domain of prostate-specific membrane antigen (PSMA). For these studies, D2B was labeled both with ${ }^{111} \mathrm{In}$ and with the NIRF dye IRDye800CW. Methods: D2B was conjugated with $N$-hydroxysuccinimide-IRDye800CW and $p$-isothiocyanatobenzyl-diethylenetriaminepentaacetic acid (ITC-DTPA) and subsequently radiolabeled with ${ }^{111} \mathrm{In}$. For biodistribution and NIRF imaging, ${ }^{111} \mathrm{In}-\mathrm{DTPA}-\mathrm{D} 2 \mathrm{~B}-\mathrm{IRD}$ ye800CW $(2 \mu \mathrm{g}$, $0.55 \mathrm{MBq} /$ mouse) was injected intravenously into BALB/c nude mice with subcutaneous PSMA-expressing LNCaP tumors (right flank) and PSMA-negative PC3 tumors (left flank). The biodistribution was determined at 1, 2, 3, and $7 \mathrm{~d}$ after injection. In addition, micro-SPECT/CT and NIRF imaging with ${ }^{111} \mathrm{In}-\mathrm{DTPA}-\mathrm{D} 2 \mathrm{~B}-$ IRDye800CW (3 $\mu \mathrm{g}, 8.5 \mathrm{MBq} /$ mouse) was performed on mice with intraperitoneally growing LS174T-PSMA tumors. Results: ${ }^{111}$ In-DTPA-D2B-IRDye800CW specifically accumulated in subcutaneous PSMA-positive LNCaP tumors $(45.8 \pm 8.0$ percentage injected dose per gram at $168 \mathrm{~h}$ after injection), whereas uptake in subcutaneous PSMA-negative PC3 tumors was significantly lower $(6.6 \pm 1.3$ percentage injected dose per gram at $168 \mathrm{~h}$ after injection). Intraperitoneal LS174T-PSMA tumors could be visualized specifically with both micro-SPECT/CT and NIRF imaging at $2 \mathrm{~d}$ after injection, and the feasibility of image-guided resection of intraperitoneal tumors was demonstrated in this model. Conclusion: Dual-labeled ${ }^{111}$ In-DTPA-D2B-IRDye800CW enables specific and sensitive detection of prostate cancer lesions in vivo with micro-SPECT/CT and NIRF imaging. In addition to preoperative micro-SPECT/CT imaging to detect tumors, NIRF imaging enables image-guided surgical resection. These preclinical findings warrant clinical studies with ${ }^{111}$ In-DTPA-D2B-IRDye800CW to improve tumor detection and resection in prostate cancer patients.

\footnotetext{
Received Jan. 29, 2014; revision accepted Feb. 19, 2014.

For correspondence or reprints contact: Susanne Lütje, Department of Nuclear Medicine-756, Radboud University Medical Center, P.O. Box 9101, 6500 HB Nijmegen, The Netherlands.

E-mail: susanne.lutje@radboudumc.nl

Published online Apr. 3, 2014.

COPYRIGHT (c) 2014 by the Society of Nuclear Medicine and Molecular Imaging, Inc.
}

Key Words: prostate cancer; PSMA; dual-modality imaging; fluorescence imaging; IRDye800CW; D2B IgG

J Nucl Med 2014; 55:995-1001

DOI: 10.2967/jnumed.114.138180

D espite improvements in diagnostic and therapeutic approaches, prostate cancer remains one of the leading causes of cancer-related death in men in the Western world. For patients with low- and intermediate-risk localized prostate cancer, and for selected patients with high-risk localized disease, surgical removal of the cancerous foci is currently regarded as the treatment option of choice (1). In tissue specimens obtained after surgical removal of localized prostate cancer during radical prostatectomy, positive surgical margins are reported in $11 \%-48 \%$ of the cases (2-4). Although the role of positive surgical margins in cancer-specific mortality remains controversial, positive surgical margins are recognized as a risk factor for prostate-specific antigen-defined biochemical recurrence (5), which is associated with the need for secondary therapy and may have a considerable impact on the patient's quality of life.

At present, several methods are clinically used to identify malignant tissues during surgery. First, cancerous tissue can be distinguished from benign structures visually on the basis of color, texture, and morphology. In addition, tactile palpation can aid the surgeon in detecting tumor tissue because of differences in elasticity or plasticity (6). However, the detection of tumor cells in resection margins and micrometastases remains extremely challenging. Therefore, imaging techniques that can aid the identification of small cancer foci are needed.

Recently, progress has been made in the field of intraoperative detection of malignant tissue with fluorescence imaging, a sensitive technique that may improve visualization of positive resection margins during surgery using fluorescent dyes that accumulate in tumor tissues. van Dam et al. (7). reported the first-in-human use of intraoperative tumor-specific fluorescence imaging for real-time visualization of tumor tissue in patients with ovarian cancer. In that study, ovarian cancer overexpressing the folate receptor was targeted and visualized with folate conjugated to the fluorescent dye fluorescein isothiocyanate.

However, a shortcoming of fluorescence imaging is the limited penetration depth of emitted light in tissue. When tumors are covered by other tissue, the fluorescent signal may not be visible and tumors may be missed. The use of near-infrared fluorescent 
(NIRF) dyes, which emit light in the near-infrared region (650$900 \mathrm{~nm}$ ), can improve the tissue penetration depth to several millimeters (8). Recently, the first clinical trial using the antibody bevacizumab tagged to the NIRF dye IRDye800CW began (clinicaltrials.gov no. NCT01508572), aiming to show the feasibility of intraoperative fluorescence imaging in breast cancer patients.

A new approach to deal with the limited tissue penetration of NIRF imaging is the combination of this technique with radionuclide detection. Both radionuclide and NIRF imaging are highly sensitive in the detection of cancer lesions. A dual-labeled targeting agent with both a radionuclide and a fluorescent moiety could be used for 3 purposes. First, the radionuclide allows preoperative assessment of the tumor burden by SPECT or PET imaging. Second, the radionuclide aids the surgeon in intraoperatively localizing the tumor using a $\gamma$ probe. Finally, the optical label precisely evaluates resection margins during surgery and detects micrometastases once the surgical field is exposed to the surface.

Because the prostate-specific membrane antigen (PSMA) is selectively overexpressed on the surface of prostate cancer cells, it represents an attractive target for antibody-guided targeting of cancer cells with radionuclides and fluorophores (9). In the present study, we applied this new imaging strategy and evaluated the tumor imaging characteristics of the newly developed anti-PSMA monoclonal antibody D2B labeled with both ${ }^{111} \mathrm{In}$ and IRDye800CW to provide proof of principle for the use of the dual-labeled imaging approach in mice with subcutaneous prostate cancer xenografts. In addition, we evaluated whether this dual-labeled D2B antibody preparation can be used to improve intraoperative visualization and to perform image-guided resection of tumors in mice with intraperitoneally growing prostate tumors.

\section{MATERIALS AND METHODS}

The anti-PSMA monoclonal antibody D2B (IgG1) (10) was purified from a hybridoma culture supernatant by protein A affinity chromatography. The antibody-secreting cells were obtained according to hybridoma technology from mice that were immunized with a cell lysate of membranes of LNCaP cells. The mice were injected 3 times (i.e., days 0,14 , and 28) intraperitoneally with $800 \mu \mathrm{g}$ of lysate and Freund adjuvant (Sigma-Aldrich). The last $3 \mathrm{~d}$ before the spleen of the mice was harvested, they were boosted with $10 \mu \mathrm{g}$ of recombinant PSMA protein (Novagen) intravenously.

\section{Mouse Tumor Models}

Male BALB/c nude mice (Janvier), 7-8 wk old, were housed in filter-topped cages (5 mice per cage) under nonsterile standard conditions with free access to standard animal chow and water. After $1 \mathrm{wk}$ of adaptation to laboratory conditions, $3 \times 10^{6}$ PSMA-expressing $\mathrm{LNCaP}$ cells were suspended in $200 \mu \mathrm{L}$ of $33 \%$ complete RPMI 1640 medium with $67 \%$ Matrigel (BD Biosciences) and injected subcutaneously (right flank). PSMA-negative PC3 cells were suspended in $200 \mu \mathrm{L}$ of $67 \%$ complete RPMI 1640 medium with 33\% Matrigel. LNCaP cells were inoculated $7 \mathrm{~d}$ before PC 3 cells, and tumors grew to approximately $0.1 \mathrm{~g}$ in 14 and $7 \mathrm{~d}$, respectively. Both cell lines were grown in RPMI 1640 medium supplemented with $10 \%$ fetal calf serum (Life Technologies) and $2 \mathrm{mM}$ glutamine.

For the intraperitoneal tumor model, LS174T colon carcinoma cells were transfected with human PSMA using the plasmid pcDNA3.1hPSMA (11). Cells were transfected using X-tremeGENE 9 DNA transfection reagent (Roche Applied Science). After transfection, single clones stably expressing PSMA were selected and grown in the presence of a $0.3 \mathrm{mg} / \mathrm{mL}$ concentration of G418. PSMA expression of
LS174T-PSMA cells was confirmed by fluorescence-activated cell sorting (FACS) analysis. LS174T-PSMA cells were grown in RPMI 1640 medium supplemented with $10 \%$ fetal calf serum and glutamine in the presence of a $0.3 \mathrm{mg} / \mathrm{mL}$ concentration of G418. LS174TPSMA cells $\left(1 \times 10^{6}\right)$ were injected intraperitoneally in $200 \mu \mathrm{L}$ of complete RPMI 1640 medium and grew for $21 \mathrm{~d}$ after inoculation.

For FACS analysis, stably transfected LS174T-PSMA cells $(150,000$ cells per well in a V-bottom 96-well plate) were washed twice and incubated on ice for $30 \mathrm{~min}$ with $25 \mu \mathrm{L}$ of either phosphate-buffered saline $(0.5 \%)$ and $5 \%$ albumin, a purified isotype-matched control antibody $(5 \mu \mathrm{g} / \mathrm{mL})$ (mouse IgG [H $+\mathrm{L}$ chain]; Jackson ImmunoResearch Europe Ltd.), or the D2B antibody at $5 \mu \mathrm{g} / \mathrm{mL}$ in phosphatebuffered saline and $0.5 \%$ bovine serum albumin. Untransfected LS174T cells were included as a negative control, and the PSMAexpressing prostate cancer cell line $\mathrm{LNCaP}$ was included as a positive control for PSMA expression. After the incubation, the cells were washed 3 times and incubated on ice for 30 min under light-protected conditions with $25 \mu \mathrm{L}$ of secondary fluorescently labeled antibody (goat-antimouse- $\mathrm{IgG}[\mathrm{H}+\mathrm{L}$ chain]-Alexa488, $2.5 \mu \mathrm{g} / \mathrm{mL}$; Life Technologies), in phosphate-buffered saline and $0.5 \%$ bovine serum albumin. The cells were washed again 3 times and measured on a FACS Calibur system with CELLQuest software (both from Beckton Dickinson Immunocytometry Systems).

All experiments were approved by the institutional Animal Welfare Committee of the Radboud University Medical Center and were conducted in accordance with the principles set forth by the Revised Dutch Act on Animal Experimentation.

\section{Synthesis of Diethylenetriaminepentaacetic Acid (DTPA)-D2B-IRDye800CW}

The murine monoclonal antibody D2B IgG $(5 \mathrm{mg})$ was conjugated with $N$-hydroxysuccinimide-IRDye $800 \mathrm{CW}$ (LI-COR Biosciences) in $2 \mathrm{~mL}$ of $0.1 \mathrm{M} \mathrm{NaHCO}_{3}, \mathrm{pH} 8.5$, with a 16-fold molar excess of $\mathrm{N}$-hydroxysuccinimide-IRDye $800 \mathrm{CW}(620 \mu \mathrm{g})$. After $2 \mathrm{~h}$ of incubation at room temperature on an orbital shaker in the dark, IRDye $800 \mathrm{CW}$ D2B was purified by gel filtration on a PD10 column eluted with 0.1 $\mathrm{M} \mathrm{NaHCO}_{3}, \mathrm{pH}$ 8.5. The conjugation ratio of the purified conjugate was determined on an Ultrospec 2000 spectrophotometer (Pharmacia Biotech) at wavelengths of $280 \mathrm{~nm}$ and $774 \mathrm{~nm}$, and the substitution ratio was calculated using the following formula: substitution ratio $=$ $0.875 \times E_{774} /\left(E_{280}-\left(0.03 \times E_{774}\right)\right)$. The substitution ratio of the conjugate used in these studies was 1.2. Subsequently, $p$-isothiocyanatobenzyl (ITC)-DTPA (Macrocyclics) was added to the conjugate in $0.1 \mathrm{M} \mathrm{NaHCO}_{3}, \mathrm{pH} 9.5(2.5 \mathrm{~mL})$, in a 27-fold molar excess (4.1 mg of IRDye800CW-D2B with $481 \mu \mathrm{g}$ of ITC-DTPA). After $1 \mathrm{~h}$ of incubation at room temperature on an orbital shaker in the dark, the reaction mixture was dialyzed for $3 \mathrm{~d}$ in a Slide-A-Lyzer (10-kDa cutoff; Pierce) against $0.25 \mathrm{M} \mathrm{NH}_{4} \mathrm{Ac}, \mathrm{pH} 5.4$.

\section{Radiolabeling of DTPA-D2B-IRDye800CW}

For biodistribution studies, DTPA-D2B-IRDye800CW $(100 \mu \mathrm{g})$ was radiolabeled with $32 \mathrm{MBq}$ of ${ }^{111} \mathrm{In}$ (Covidien) in $0.1 \mathrm{M} 2-(\mathrm{N}-$ morpholino)ethanesulfonic acid buffer, $\mathrm{pH} 5.4$ (3 times the volume of ${ }^{111}$ In-chloride), and incubated for $30 \mathrm{~min}$ at room temperature under metal-free conditions. For SPECT/CT studies, DTPA-D2B-IRDye800CW $(35 \mu \mathrm{g})$ was incubated with $280 \mathrm{MBq}$ of ${ }^{111} \mathrm{In}$ in $0.1 \mathrm{M}$ maintenance electrolyte solution buffer, $\mathrm{pH}$ 5.4. After incubation, $50 \mathrm{mM}$ ethylenediaminetetraacetic acid was added to the final concentration of $5 \mathrm{mM}$ to chelate unincorporated ${ }^{111} \mathrm{In}$.

The labeling efficiency was determined by instant thin-layer chromatography using $0.15 \mathrm{M}$ citrate buffer, $\mathrm{pH} 6.0$, as the mobile phase. The labeling efficiency was $85 \%$ for the biodistribution studies and $36 \%$ for the micro-SPECT/CT study. ${ }^{111}$ In-DTPA-D2B-IRDye $800 \mathrm{CW}$ was purified by gel filtration on a PD-10 column, and the radiochemical 


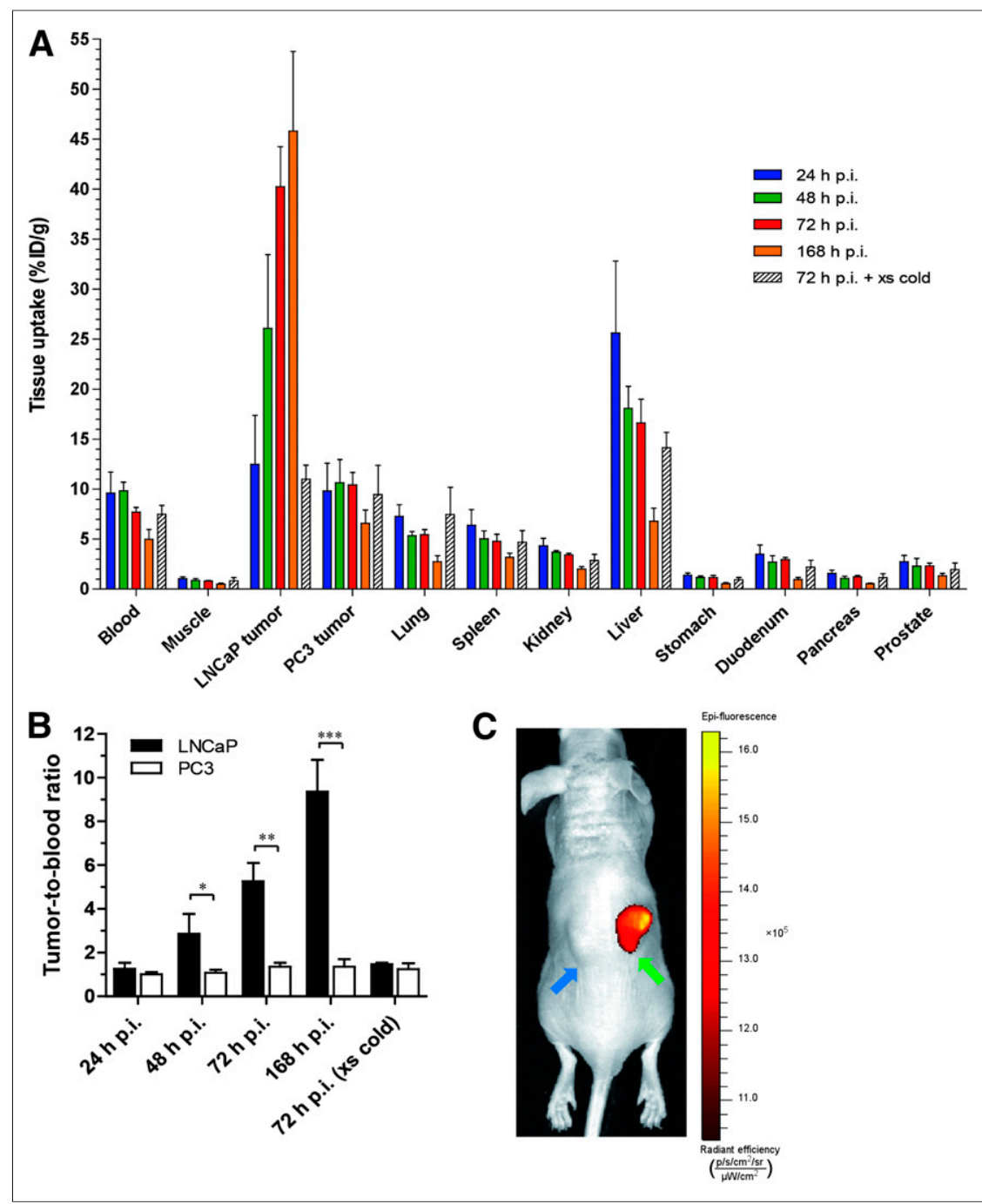

FIGURE 1. (A) Biodistribution of ${ }^{111}$ In-DTPA-D2B-IRDye800CW (0.55 MBq, $\left.2.0 \mu \mathrm{g} / \mathrm{mouse}\right)$ in mice with subcutaneous PSMA-positive LNCaP and PSMA-negative PC3 xenografts at several time points after injection. Excess of unlabeled D2B $\operatorname{lgG}(300 \mu \mathrm{g} / \mathrm{mouse})$ was coinjected in one group of mice as negative control. LNCaP-to-PC3 tumor ratios at $48 \mathrm{~h}$ after injection were $2.5 \pm$ 0.7. (B) PSMA-positive and PSMA-negative tumor-to-blood ratios at several time points after injection of ${ }^{111}$ In-DTPA-D2B-IRDye800CW. (C) For illustration purposes, NIRF image of a mouse bearing a PSMA-positive LNCaP tumor on right flank (green arrow) and a PSMA-negative PC3 tumor on the left flank (blue arrow) at $48 \mathrm{~h}$ after intravenous injection of dual-labeled ${ }^{111} \mathrm{In}$-DTPAD2B-IRDye800CW. ${ }^{\star} P<0.0001$ on $t$ testing. ${ }^{\star \star} P<0.0001$ on $t$ testing. ${ }^{\star \star \star} P<0.0001$ on $t$ testing. $\mathrm{p}=$ photons; p.i. $=$ after injection; $\mathrm{sr}=$ steradian; $\mathrm{xs}=$ excess.

purity of the final dual-labeled tracer was at least $97 \%$, which was determined by instant thin-layer chromatography.

\section{Immunoreactivity}

The immunoreactive fraction of the dual-labeled ${ }^{111}$ In-DTPA-D2BIRDye800CW was compared with that of ${ }^{111}$ In-DTPA-D2B using freshly trypsinized LNCaP cells, essentially as described by Lindmo et al. and Heskamp et al. $(12,13)$. Briefly, a serial dilution of LNCaP cells $\left(6.3 \times 10^{5}\right.$ to $1.0 \times 10^{6}$ cells $\left./ \mathrm{mL}\right)$ in $0.5 \mathrm{~mL}$ of RPMI medium containing $0.5 \%$ bovine serum albumin was incubated at $37^{\circ} \mathrm{C}$ for $1 \mathrm{~h}$ with $0.002 \mathrm{pmol}$ of ${ }^{111} \mathrm{In}-\mathrm{DTPA}-\mathrm{D} 2 \mathrm{~B}-\mathrm{IRD}$ ye800CW (350 Bq). To determine nonspecific binding, an excess of unlabeled DTPA-D2BIRDye $800 \mathrm{CW}(160 \mathrm{pmol})$ was added to a duplicate of the lowest cell concentration. After incubation, the cells were centrifuged and washed with $500 \mu \mathrm{L}$ of RPMI medium containing $0.5 \%$ bovine serum albu- min. The activity in the vials and in the cell pellet was determined in the $\gamma$ counter (Wizard 3" 1480; LKB-Wallac). The inverse of the specific cell-bound activity was plotted against the inverse of the cell concentration, and the immunoreactive fraction was calculated from the $y$-axis intercept using GraphPad Prism software (version 5.03 for Windows [Microsoft]; GraphPad Software).

\section{Competitive Binding Assay}

The inhibitory concentration of $50 \%$ for D2B IgG was determined in a competitive binding assay on $\mathrm{LNCaP}$ cells using ${ }^{111} \mathrm{In}$ labeled D2B as a tracer. $\mathrm{LNCaP}$ cells were grown to confluency in 6-well plates and incubated on ice for $2 \mathrm{~h}$ in $1 \mathrm{~mL}$ of binding buffer with $1.85 \mathrm{kBq}$ of ${ }^{111}$ In-labeled D2B IgG and increasing concentrations (0.005$300 \mathrm{nM}$ ) of unlabeled D2B IgG. Cells were washed with binding buffer after incubation, and the cell-associated activity was measured in a $\gamma$ counter. Inhibitory concentrations of $50 \%$ were calculated using Prism software, version 5.03 .

\section{Biodistribution and NIRF Imaging in Mice with Subcutaneous Tumors}

For biodistribution studies, 4 groups of male $\mathrm{BALB} / \mathrm{c}$ nude mice bearing LNCaP (right flank) and PC3 (left flank) xenografts were injected into the tail vein with the dual-labeled conjugate ${ }^{111} \mathrm{In}$-DTPA-D2BIRDye 800CW (0.55 MBq, $2.0 \mu \mathrm{g} /$ mouse). Three additional mice (control group) were coinjected intravenously with an excess of unlabeled D2B IgG (300 $\mu \mathrm{g} /$ mouse) to determine nonspecific uptake of ${ }^{111}$ In-DTPAD2B-IRDye $800 \mathrm{CW}$.

At 24, 48, and $72 \mathrm{~h}$ after injection, 4 mice per time point $(5$ mice at $168 \mathrm{~h}$ after injection) were euthanized with $\mathrm{CO}_{2} / \mathrm{O}_{2}$ asphyxiation and tissues of interest ( $\mathrm{LNCaP}$ and PC3 tumors, muscle, lung, spleen, kidney, liver, pancreas, stomach, duodenum, and prostate) were dissected and weighed and the radioactivity was measured in a $\gamma$ counter. Blood samples were obtained by heart puncture. For calculation of radioactivity uptake in each tissue as a fraction of the injected dose, an aliquot of the injected dose was counted simultaneously.

The mice that were scheduled for biodistribution studies at $48 \mathrm{~h}$ after injection were imaged with an in vivo imaging system (Xenogen VivoVision IVIS Lumina II [Caliper Life Sciences]; acquisition time, $5 \mathrm{~min}$ ) before dissection.

The control group of mice that received an excess of unlabeled D2B IgG in addition to intravenously injected ${ }^{111}$ In-DTPA-D2BIRDye $800 \mathrm{CW}$ was euthanatized and dissected as described above at $72 \mathrm{~h}$ after injection.

\section{Dual-Modality Micro-SPECT/CT and NIRF Imaging and Image-Guided Surgery}

Four male BALB/c nude mice with intraperitoneally growing LS174T-PSMA tumors were intravenously injected with the dual- 


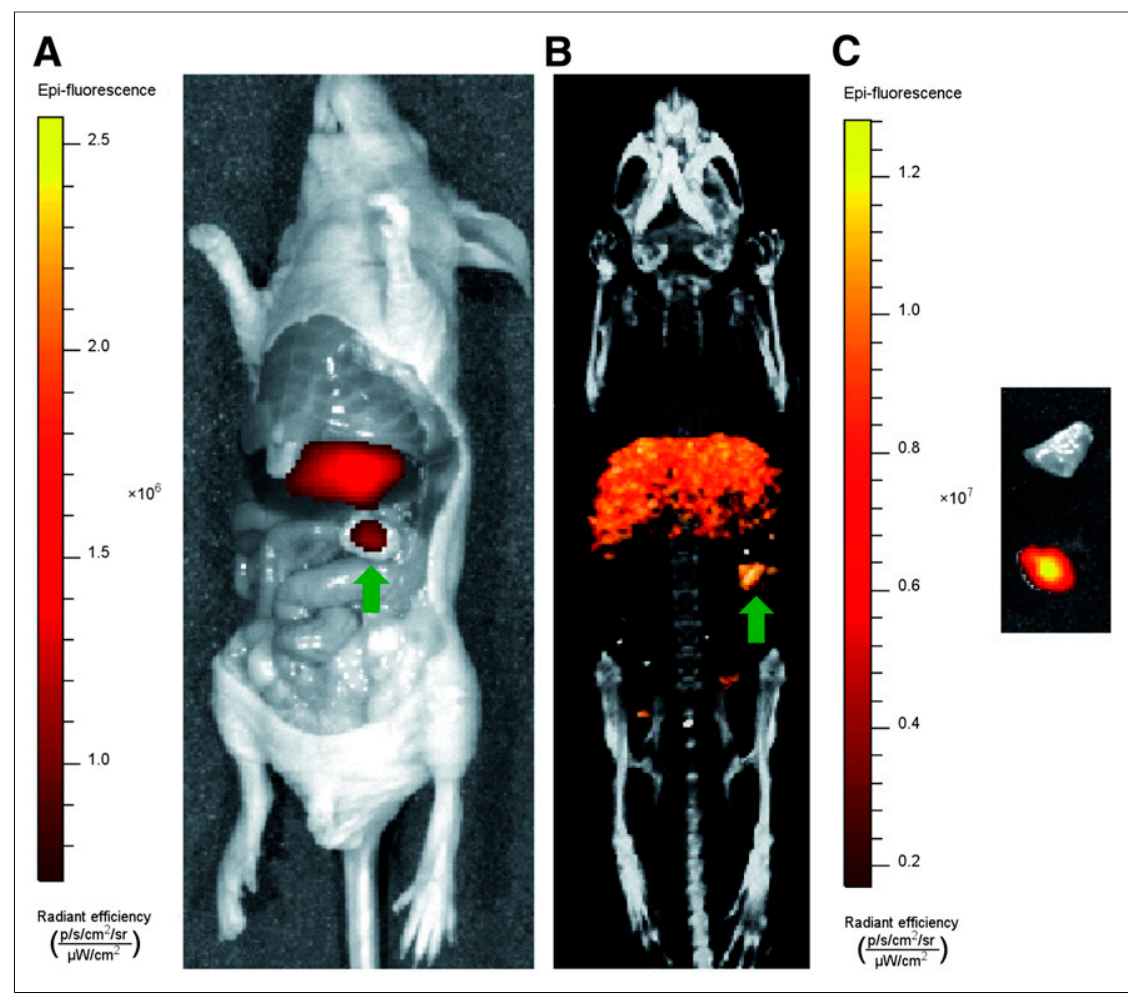

FIGURE 2. Dual-modality fluorescence and micro-SPECT/CT imaging with dual-labeled ${ }^{111} \mathrm{In}$ DTPA-D2B-IRDye800CW at $48 \mathrm{~h}$ after injection in a mouse with an intraperitoneally growing LS174T-PSMA tumor. Shown are NIRF image (acquisition time, $5 \mathrm{~min}$ ) (A), corresponding microSPECT/CT image with mouse supine (B), and NIRF image of muscle sample (top) and tumor (bottom) after resection (C). Green arrows indicate the PSMA-positive tumor. $\mathrm{p}=$ photons; $\mathrm{sr}=$ steradian.

labeled ${ }^{111}$ In-DTPA-D2B-IRDye800CW (8.5 MBq and $3.0 \mu \mathrm{g} /$ mouse) and imaged at $48 \mathrm{~h}$ after injection on both the in vivo imaging system (Xenogen VivoVision IVIS Lumina II; acquisition time, $5 \mathrm{~min}$ ) and a small-animal micro-SPECT/CT scanner (U-SPECT II; MILabs) with a 1.0-mm-diameter pinhole collimator tube (acquisition time, $30 \mathrm{~min}$ ). Micro-SPECT/CT was performed preoperatively, followed by NIRF imaging of the mice in supine position after surgical removal of skin, abdominal muscle layers, and peritoneum. After in vivo image acquisition, the visualized tumors were resected, followed by NIRF imaging to control whether residual tumor tissue was in situ. In addition, micro-SPECT/CT was performed after resection of the tumors using the same scanning parameters that were used for preoperative evaluation. Scans were reconstructed with MILabs reconstruction software, which uses an ordered-subset expectation maximization algorithm.

\section{Statistical Analysis}

Statistical analyses were performed with Prism, version 5.03. Results are presented as mean \pm SD.

\section{RESULTS}

\section{In Vitro Characterization}

The immunoreactive fractions of ${ }^{111}$ In-DTPA-D2B-IRDye800CW and ${ }^{111}$ In-DTPA-D2B were $75 \%$ and $84 \%$, respectively, indicating that the immunoreactivity of the D2B antibody was largely preserved during the 2 conjugation procedures.

The inhibitory concentration of $50 \%$ for D2B IgG was $3.7 \mathrm{nM}$ (95\% confidence interval, $2.8-4.8 \mathrm{nM}$ ).
Biodistribution Studies and Imaging of Subcutaneous Tumors

${ }^{111}$ In-DTPA-D2B-IRDye800CW specifically accumulated in the subcutaneous PSMA-positive LNCaP tumors, reaching $12.5 \pm 4.9$ percentage injected dose per gram $(\% \mathrm{ID} / \mathrm{g})(n=4)$ at $24 \mathrm{~h}$ after injection, $26.1 \pm 7.4 \% \mathrm{ID} / \mathrm{g}(n=4)$ at $48 \mathrm{~h}$ after injection, $40.2 \pm 4.0 \% \mathrm{ID} / \mathrm{g}(n=4)$ at $72 \mathrm{~h}$ after injection, and $45.8 \pm 8.0 \%$ $\mathrm{ID} / \mathrm{g}(n=5)$ at $168 \mathrm{~h}$ after injection (Fig. 1A). Tumor uptake in PSMA-negative PC3 xenografts was significantly lower at 48, 72, and $168 \mathrm{~h}$ after injection, reaching $9.8 \pm$ $2.8 \% \mathrm{ID} / \mathrm{g}(n=4)$ at $24 \mathrm{~h}$ after injection $(P=0.3768), 10.6 \pm 2.3 \% \mathrm{ID} / \mathrm{g}(n=4)$ at $48 \mathrm{~h}$ after injection $(P=0.0071), 10.4 \pm$ $1.2 \% \mathrm{ID} / \mathrm{g}(n=4)$ at $72 \mathrm{~h}$ after injection $(P<0.0001)$, and $6.6 \pm 1.3 \% \mathrm{ID} / \mathrm{g}(n=5)$ at $168 \mathrm{~h}$ after injection $(P<0.0001)$.

The uptake of ${ }^{111}$ In-DTPA-D2BIRDye $800 \mathrm{CW}$ remained low in most normal tissues; uptake in the liver ranged from $25.6 \pm 7.2 \% \mathrm{ID} / \mathrm{g}$ at $24 \mathrm{~h}$ after injection to $6.8 \pm 1.3 \% \mathrm{ID} / \mathrm{g}$ at $168 \mathrm{~h}$ after injection (Fig. 1A).

Tumor-to-blood ratios of PSMA-positive LNCaP xenografts were $1.3 \pm 0.3$ at $24 \mathrm{~h}$ after injection, $2.9 \pm 0.9$ at $48 \mathrm{~h}$ after injection, $5.3 \pm 0.8$ at $72 \mathrm{~h}$ after injection, and $9.4 \pm 1.5$ at $168 \mathrm{~h}$ after injection. Tumor-toblood ratios of the PSMA-negative PC3 xenografts were significantly lower at 48 , 72 , and $168 \mathrm{~h}$ after injection $(P<0.0001)$, reaching $1.0 \pm 0.1$ at $24 \mathrm{~h}$ after injection, $1.1 \pm 0.1$ at $48 \mathrm{~h}$ after injection, $1.4 \pm 0.2$ at $72 \mathrm{~h}$ after injection, and $1.4 \pm 0.3$ at $168 \mathrm{~h}$ after injection (Fig. 1B). PSMA-positive-to-PSMA-negative tumor ratios were $1.2 \pm$ 0.2 at $24 \mathrm{~h}$ after injection, $2.5 \pm 0.7$ at $48 \mathrm{~h}$ after injection, $3.9 \pm$ 0.7 at $72 \mathrm{~h}$ after injection, and $7.2 \pm 2.0$ at $168 \mathrm{~h}$ after injection.

At $48 \mathrm{~h}$ after injection, an accumulation of ${ }^{111}$ In-DTPA-D2BIRDye $800 \mathrm{CW}$ could be visualized in subcutaneous PSMA-positive LNCaP tumors using the in vivo fluorescence imager, whereas PSMA-negative tumors showed no fluorescent signal (Fig. 1C).

Tumor uptake of ${ }^{111}$ In-DTPA-D2B-IRDye $800 \mathrm{CW}$ could be blocked by coinjection of an excess of $300 \mu \mathrm{g}$ of unlabeled D2B $\mathrm{IgG}$, resulting in nonspecific (non-PSMA-mediated) uptake of $11.0 \pm$ $1.41 \% \mathrm{ID} / \mathrm{g}(n=3)$ and $9.5 \pm 2.9 \% \mathrm{ID} / \mathrm{g}(n=3)$ at $72 \mathrm{~h}$ after injection in LNCaP and PC3 xenografts, respectively. This further confirms the specific uptake of ${ }^{111}$ In-DTPA-D2B-IRDye $800 \mathrm{CW}$ in the PSMA-expressing tumor.

\section{Dual-Modality Micro-SPECT/CT and NIRF Imaging in Mice with Intraperitoneal Tumors}

Five BALB/c nude mice with intraperitoneal LS174T-PSMA tumors were imaged with both the in vivo fluorescence scanner and the micro-SPECT/CT scanner at $48 \mathrm{~h}$ after injection of duallabeled ${ }^{111}$ In-DTPA-D2B-IRDye800CW. A typical set of microSPECT/CT acquisitions with corresponding fluorescence images in supine and lateral positions is shown in Figure 2. LS174TPSMA tumors were specifically visualized by both imaging modalities. When tumors were located deeper in the peritoneal cavity, 
the fluorescent signals detected were less intense or even absent because of tissue absorption of the emitted photons (Fig. 3).

\section{Image-Guided Resection of Intraperitoneal Tumors}

After preoperative evaluation of tumors by micro-SPECT/CT, image-guided resection of the tumors was performed on mice with intraperitoneally growing LS174T-PSMA nodules. First, the exact tumor location was identified by NIRF imaging. Subsequently, all detected tumors were resected and additional NIRF and microSPECT/CT imaging was performed to ensure radical surgical resection. In Figure 4, the applicability of the dual-modality probe for intraoperative image-guided resection of the intraperitoneal tumors is shown.

\section{DISCUSSION}

This study provided proof of principle for targeting PSMAexpressing prostate cancer in vivo with the dual-labeled ${ }^{111}$ InDTPA-D2B-IRDye800CW in a prostate cancer model with subcutaneous and intraperitoneal tumors. In addition, dual-modality image-guided surgery based on this dual-labeled conjugate was performed.

During surgery, detection of small cancer foci or positive surgical margins remains challenging and may be enhanced by NIRF imaging, which allows real-time intraoperative visualization of malignant tissues (7). Nontargeted NIRF imaging based on the application of fluorescent nanoparticles is frequently used for sentinel lymph node detection in patients with various malignancies (14). The first clinical proof-of-concept studies using this sentinel lymph node mapping technique with dual-modality nanocolloids showed promising results in prostate cancer (15). However, nontargeted agents are not tumor-selective and hence cannot be used for real-time visualization of tumor cells in resection margins. The use of targeted imaging approaches could allow more sensitive and real-time detection of cancerous foci and positive surgical margins in the submillimeter range and would permit patient-tailored surgical interventions. Several groups so far have reported the use of fluorophores conjugated to monoclonal antibodies targeting receptors such as the epidermal growth factor receptor (16), HER2/neu receptor (17), and vascular endothelial growth factor (18).

Targeted NIRF imaging to identify prostate cancer cells in intraoperative settings using PSMA as a target was introduced by Liu et al. (19), who conjugated the low-molecular-weight PSMA ligand CTT-54.2 with Cy5.5 and demonstrated the tumor-binding potential of the Cy5.5-CTT-54.2 conjugate in vitro. Recently, the humanized anti-PSMA monoclonal antibody J591 was conjugated with an indocyanine green derivative by Nakajima et al. This conjugate showed specific targeting of the subcutaneous PSMAexpressing tumors in mice (20). Chen et al. (21) conjugated the anti-PSMA monoclonal antibody YC-27 with IRDye800CW. In mice with subcutaneous PC3-PIP tumors, tumor-to-nontarget ratios of 10 were obtained. Recently, Banerjee et al. synthesized the first dual-modality SPECT/NIRF imaging agent with affinity for the center of PSMA based on conjugation of a glutamate urea compound with both DOTA and IRDye800CW for targeting PSMAexpressing prostate cancer in a subcutaneous mouse model (22).

In the present study, we showed that NIRF-SPECT dual-modality imaging allows specific visualization of both subcutaneous and intraperitoneal prostate cancer tumors, providing proof of principle for image-guided surgery with ${ }^{111}$ In-DTPA-D2B-IRDye800CW in mice with intraperitoneally growing prostate tumors.

Specific accumulation of ${ }^{111}$ In-DTPAD2B-IRDye800CW in the PSMA-expressing subcutaneous and intraperitoneal tumors was observed, whereas uptake in the other tissues remained low, except for the liver. Enhanced hepatic uptake of IRDye800CWconjugated antibodies has been reported in previous studies. Cohen et al. (23) reported enhanced liver uptake of the dual-labeled antibodies ${ }^{89} \mathrm{Zr}$-cetuximab-IRDye800CW and ${ }^{89} \mathrm{Zr}$-bevacizumab-IRDye800CW in vivo, which was attributed to the lipophilicity and charge of the antibody conjugate. In this study, a substitution ratio of less than 2 was found to be optimal to prevent enhanced blood clearance and hepatic liver uptake.

All subcutaneous and intraperitoneal LS174T-

PSMA tumors were clearly visualized with
FIGURE 3. Dual-modality fluorescence and micro-SPECT/CT imaging with dual-labeled ${ }^{111} \mathrm{In}-$ DTPA-D2B-IRDye800CW at $48 \mathrm{~h}$ after injection in a mouse with several intraperitoneal LS174TPSMA tumors located at different depths in the peritoneal cavity. NIRF image (acquisition time, $5 \mathrm{~min})(\mathrm{A})$ and corresponding micro-SPECT/CT images in supine (B) and left lateral (C) positions show limited penetration depth of NIRF imaging. Arrows indicate 2 tumors superficial enough to be visualized with fluorescence imaging. Tumors deeper inside peritoneal cavity are not visualized. 


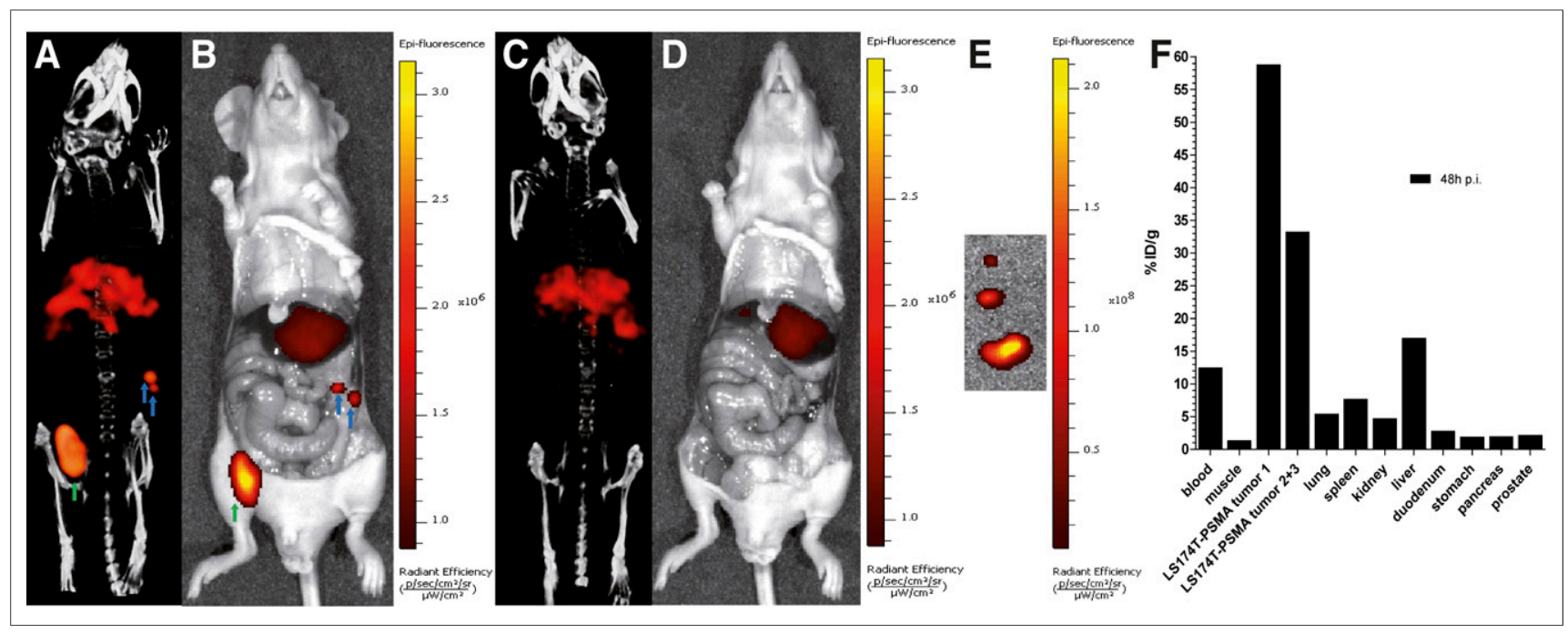

FIGURE 4. Dual-modality fluorescence and micro-SPECT/CT imaging with dual-labeled ${ }^{111} \mathrm{In}-\mathrm{DTPA}-\mathrm{D} 2 \mathrm{~B}-\mathrm{IRDye} 800 \mathrm{CW}$ at $48 \mathrm{~h}$ after injection in mouse with intraperitoneally growing LS174T-PSMA tumors. Shown are micro-SPECT/CT image of 3 intraperitoneal PSMA-positive tumors (green and blue arrows) (A), merged photograph and NIRF image of mouse before resection of 3 tumors (B), micro-SPECT/CT image after resection of tumors (C), merged photograph and NIRF image of mouse after resection of tumors (D), merged photograph and optical fluorescence image of tumors after resection (E), and biodistribution of ${ }^{111}$ In-DTPA-D2B-IRDye800CW in mice with intraperitoneal LS174T-PSMA tumors (F). $p=$ photons; p.i. = after injection; $\mathrm{sr}=$ steradian.

micro-SPECT/CT. On the corresponding in vivo fluorescence scans in an intraoperative setting, all intraperitoneal tumors that were located superficially could be visualized. However, tumors located deeper inside the peritoneal cavity may not be visualized, because of absorption of the emitted fluorescent light in tissues. Although the absorption coefficient of biologic tissue is relatively low in the near-infrared region (700-900 $\mathrm{nm}$ ), light of this wavelength can penetrate only up to a few millimeters in tissue (24). Therefore, in addition to preoperative tumor localization, dual-labeled NIRF and radionuclide targeting agents allow intraoperative mapping of more deeply lying malignant tissue with a $\gamma$ probe. Subsequently, the exact position can be determined with NIRF imaging, indicating the additive value of dual-modality imaging for detection of tumors, their resection, and real-time in vivo margin assessment.

Taken together, we showed that PSMA-expressing prostate cancer can be visualized sensitively and specifically with dual-modality micro-SPECT/CT and NIRF imaging with the newly developed ${ }^{111}$ In-DTPA-D2B-IRDye800CW conjugate in mice with subcutaneous and intraperitoneal prostate cancer xenografts. For translation of dual-modality imaging of ${ }^{111}$ In-DTPA-D2B-IRDye800CW into the clinic, this antibody construct has to be considered a new medicinal product, which has to be studied extensively regarding toxicologic aspects before it can be tested.

A major advantage for clinical use is the integration of both SPECT/CT and NIRF imaging modalities in intraoperative settings to improve image-guided surgery, which could be applied for both open laparotomy and endoscopic procedures.

\section{CONCLUSION}

Here, we have demonstrated the feasibility of targeting PSMAexpressing prostate cancer with the dual-labeled ${ }^{111}$ In-DTPAD2B-IRDye800CW imaging agent and provided proof of principle that dual-modality image-guided surgery using this conjugate is feasible. Prostate cancer lesions were specifically and sensitively detected in vivo in a mouse model with intraperitoneal PSMA- expressing tumors. In this setting, radionuclide imaging may allow preoperative detection and intraoperative localization of tumors, whereas NIRF imaging enables subsequent accurate delineation of tumors and real-time assessment of resection margins. These preclinical findings encourage future clinical studies with targeted duallabeled imaging probes for pre- and intraoperative guidance of prostate tumor resection, to achieve radical removal of tumor tissue.

\section{DISCLOSURE}

The costs of publication of this article were defrayed in part by the payment of page charges. Therefore, and solely to indicate this fact, this article is hereby marked "advertisement" in accordance with 18 USC section 1734 . This study was performed within the framework of CTMM, the Center for Translational Molecular Medicine; the PCMM project (grant 03O-203); and the Dutch Cancer Society (grant KUN-2010-4820). This work was supported by Fondazione Cariverona (Verona Nanomedicine Initiative and Interceptin project), AIRC 5x1000, and Fondazione Cariverona/AIRC Progetto Regionale. No other potential conflict of interest relevant to this article was reported.

\section{ACKNOWLEDGMENTS}

We thank Bianca Lemmers-van de Weem, Henk Arnts, Iris LamersElemans, and Kitty Lemmens-Hermans for their excellent technical assistance in the animal experiments.

\section{REFERENCES}

1. Heidenreich A, Bellmunt J, Bolla M, et al.; European Association of Urology. EAU guidelines on prostate cancer. Part 1: screening, diagnosis, and treatment of clinically localised disease. Eur Urol. 2011;59:61-71.

2. Eastham JA, Kuroiwa K, Ohori M, et al. Prognostic significance of location of positive margins in radical prostatectomy specimens. Urology. 2007;70:965-969.

3. Eastham JA, Kattan MW, Riedel E, et al. Variations among individual surgeons in the rate of positive surgical margins in radical prostatectomy specimens. J Urol. 2003;170:2292-2295. 
4. Yossepowitch O, Briganti A, Eastham JA, et al. Positive surgical margins after radical prostatectomy: a systematic review and contemporary update. Eur Urol. 2014;65:303-313.

5. Stephenson AJ, Eggener SE, Hernandez AV, et al. Do margins matter? The influence of positive surgical margins on prostate cancer-specific mortality. Eur Urol. 2014;65:675-680.

6. Kelderhouse LE, Chelvam V, Wayua C, et al. Development of tumor-targeted near infrared probes for fluorescence guided surgery. Bioconjug Chem. 2013;24:1075-1080.

7. van Dam GM, Themelis G, Crane LM, et al. Intraoperative tumor-specific fluorescence imaging in ovarian cancer by folate receptor- $\alpha$ targeting: first in-human results. Nat Med. 2011;17:1315-1319.

8. Fomina N, McFearin CL, Sermsakdi M, Morachis JM, Almutairi A. Low power, biologically benign NIR light triggers polymer disassembly. Macromolecules. 2011;44:8590-8597.

9. Ross JS, Sheehan CE, Fisher HA, et al. Correlation of primary tumor prostatespecific membrane antigen expression with disease recurrence in prostate cancer. Clin Cancer Res. 2003;9:6357-6362.

10. Colombatti M, Fracasso G, Cingarlini S, Canevari S, Figini M, inventors; Università Degli Studi Di Verona, Fondazione IRCCS "Istituto Nazionale Dei Tumori", assignee. Isolated monoclonal antibody or fragment thereof binding prostate specific membrane antigen, conjugates and uses thereof. International Patent Application PCT/IB2009/005326. October 29, 2009.

11. Castelletti D, Fracasso G, Alfalah M, Cingarlini S, Colombatti M, Naim HY. Apical transport and folding of prostate-specific membrane antigen occurs independent of glycan processing. J Biol Chem. 2006;281:3505-3512.

12. Lindmo T, Boven E, Cuttitta F, Fedorko J, Bunn PA Jr. Determination of the immunoreactive fraction of radiolabeled monoclonal antibodies by linear extrapolation to binding at infinite antigen excess. J Immunol Methods. 1984;72:77-89.

13. Heskamp S, van Laarhoven HW, Molkenboer-Kuenen JD, et al. ImmunoSPECT and immunoPET of IGF-1R expression with the radiolabeled antibody R1507 in a triple-negative breast cancer model. J Nucl Med. 2010;51:1565-1572.

14. van den Berg NS, Valdés-Olmos RA, van der Poel HG, van Leeuwen FW. Sentinel lymph node biopsy for prostate cancer: a hybrid approach. J Nucl Med. 2013;54:493-496.
15. van der Poel HG, Buckle T, Brouwer OR, Valdés Olmos RA, van Leeuwen FW. Intraoperative laparoscopic fluorescence guidance to the sentinel lymph node in prostate cancer patients: clinical proof of concept of an integrated functional imaging approach using a multimodal tracer. Eur Urol. 2011;60:826-833.

16. Gleysteen JP, Duncan RD, Magnuson JS, Skipper JB, Zinn K, Rosenthal EL. Fluorescently labeled cetuximab to evaluate head and neck cancer response to treatment. Cancer Biol Ther. 2007;6:1181-1185.

17. Lee SB, Hassan M, Fisher R, et al. Affibody molecules for in vivo characterization of HER2-positive tumors by near-infrared imaging. Clin Cancer Res. 2008;14:3840-3849.

18. Withrow KP, Newman JR, Skipper JB, et al. Assessment of bevacizumab conjugated to Cy5.5 for detection of head and neck cancer xenografts. Technol Cancer Res Treat. 2008;7:61-66.

19. Liu T, Wu LY, Hopkins MR, Choi JK, Berkman CE. A targeted low molecular weight near-infrared fluorescent probe for prostate cancer. Bioorg Med Chem Lett. 2010;20:7124-7126.

20. Nakajima T, Mitsunaga M, Bander NH, Heston WD, Choyke PL, Kobayashi H. Targeted, activatable, in vivo fluorescence imaging of prostate-specific membrane antigen (PSMA) positive tumors using the quenched humanized J591 antibodyindocyanine green (ICG) conjugate. Bioconjug Chem. 2011;22:1700-1705.

21. Chen Y, Dhara S, Banerjee SR, et al. A low molecular weight PSMA-based fluorescent imaging agent for cancer. Biochem Biophys Res Commun. 2009;390: $624-629$.

22. Banerjee SR, Pullambhatla M, Byun Y, et al. Sequential SPECT and optical imaging of experimental models of prostate cancer with a dual modality inhibitor of the prostate-specific membrane antigen. Angew Chem Int Ed Engl. 2011;50: 9167-9170.

23. Cohen R, Stammes MA, de Roos IH, Stigter-van Walsum M, Visser GW, van Dongen GA. Inert coupling of IRDye800CW to monoclonal antibodies for clinical optical imaging of tumor targets. EJNMMI Res. 2011;1:31.

24. Kovar JL, Simpson MA, Schutz-Geschwender A, Olive DM. A systematic approach to the development of fluorescent contrast agents for optical imaging of mouse cancer models. Anal Biochem. 2007;367:1-12. 